\title{
Exploring Futures for Particle Physics
}

\section{Particle physicists in the US are embarking on a year-long community study to examine options for the future landscape of their field.}

\author{
By Chris Quigg
}

A II scientists look to the future, on timescales from immediate to long-term. Particle physicists are no different, but their view is colored by projects that span decades, instruments that require large investments, and research teams that range from a few individuals to thousands. Accordingly, the global particle physics community has a special need for inclusive, coherent planning. Over the next year, US particle physicists, joined by international colleagues, will engage in an in-depth community study, Snowmass 2021, to define the most important questions for the field and to identify the most promising opportunities to address these questions in a global context.

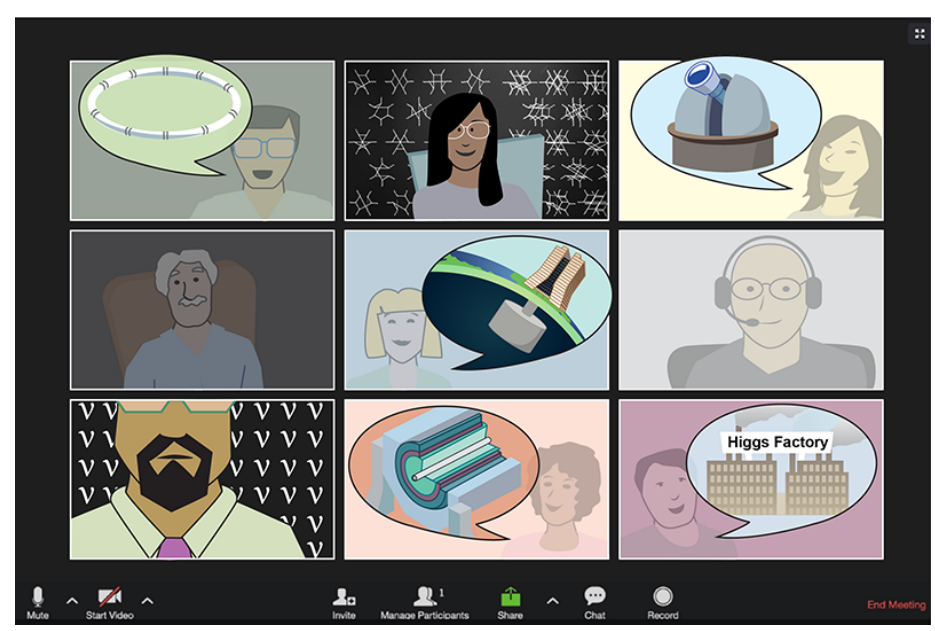

Snowmass 2021 will bring together (virtually) a wide variety of particle physicists to discuss future projects, such as colliders, underground experiments, astronomical observatories, and "Higgs factories."

Credit: APS/Carin Cain
The name of this community study derives from a particle physics meeting at Snowmass, Colorado, organized in the summer of 1982 by the Division of Particles and Fields of the American Physical Society. That gathering was the first modern attempt to bring an eclectic group of active researchers together to consider an optimal national program. Snowmass 2021 is gearing up now with a community planning meeting on October 5-8. In my view, the first goal of this study should be to expand the horizons of individual physicists and the field-to look beyond our current research problems, our collaborations, our institutions, and to learn from others. I would like to see every initiative emerge from Snowmass better understood, improved, and taken more seriously, so that new possibilities can take shape in all subdisciplines. Then we can move forward to set priorities, which will eventually be recommended to funding agencies through the High Energy Physics Advisory Panel.

Snowmass follows a similar European study that spanned nearly two years. Informed by that effort, the CERN Council unanimously decided in June of this year to update the European Strategy for Particle Physics, setting priorities for European ambitions in a worldwide context and taking account of developments in neighboring disciplines [1]. The update identifies the detailed exploration of the Higgs boson as the most pressing priority for the field. Projecting what will be known when the Large Hadron Collider (LHC) ceases operation in 2038, it cites an electron-positron collider "Higgs factory" as the most appropriate new instrument to advance that agenda. The Eurostrategy further recommends an assessment of the technical and financial feasibility of a $100-\mathrm{TeV}$ proton-proton collider, seen as the most powerful instrument for exploring new scientific terrain. 
Snowmass 2021 will certainly consider future accelerators, as well as many other projects that interest particle physicists and their close scientific neighbors. The technical aspects can be summarized in a few queries. What are the most important, and timely, issues for our science? Which instruments-accelerators, detectors, observatories-offer the best potential to achieve these objectives? What technologies must we develop to build these instruments? There is no shortage of engaging questions, from the highly specific to the marginally metaphysical, to which we would love to know the answers. I have treated some of them in a brief essay [2], and at greater length in seminars [3], where I posed 120 questions.

Many burning questions are linked to the phenomenon of electroweak symmetry breaking and the Higgs boson, which was detected in 2012 at the LHC. In the short term, we want to know more precisely that new particle's properties, to see how closely they match the electroweak theory's expectations, and to learn whether other such particles exist. This imperative will set performance goals for a Higgs factory, to be investigated at Snowmass. We must go to higher energies than a Higgs factory or the LHC can provide to study how the Higgs boson interacts with itself and whether it alone keeps weak-boson interactions from growing too rapidly at high energies. What kind of future machine and what experimental conditions could guarantee definitive answers is another important question for Snowmass.

Beyond these questions, we don't know what the next important energy scale will be. Looking upward, toward a few $\mathrm{TeV}$ and beyond, we seek new forces and new kinds of particles; we look for evidence of spacetime dimensions beyond the familiar $3+1$; and we ask what separates the electroweak scale from a unification scale or the Planck scale. There is a low-energy frontier too. Searches for axions, particle dark matter, and other feebly interacting particles press us to explore very small energy scales with new acuity.

Flavor physics, the relationship among different quarks and leptons, is immensely rich. The problem of identity-what makes an electron an electron and a top quark a top quark-is so simple to state, but we haven't yet found a promising theoretical approach to the diverse character of the constituents of matter. Neutrinos, in particular, are awash in mysteries: what is the origin of their mass, are they their own antiparticles, can we detect the cosmic neutrino background?
Other topics-quantum chromodynamics and the strong interactions, the special status of the top quark as the heaviest constituent, unified theories of the strong, weak, and electromagnetic interactions, precision measurements using techniques of atomic and molecular physics-all present many opportunities. Links with astroparticle physics, cosmology, and gravitational physics are also growing in intensity. This abundance of scientific topics calls for a national program that is diverse both in scope-by engaging a wide variety of questions-and in scale-by conducting experiments from modest to gargantuan. How can we arrive at an optimal mix?

This brief rundown gives a hint of the scientific issues to be examined, without sampling the instruments and technologies. Let me close by mentioning topics that are not strictly technical but merit attention in a community study. How can we best welcome and nurture the next generation of physicists, reflecting the diversity of the human family? How can we offer young physicists the opportunity to make their own decisions-even their own mistakes? How can we provide secure career paths for colleagues who design, build, and operate accelerators and detectors or who create computer simulations? How can we effectively nurture both theoretical research that engages with experiment and exploratory theory that doesn't yet speak with experiment? How can we make common cause with other disciplines, within physics and beyond, to strengthen the whole scientific enterprise and secure the place of science in our society?

I hope, too, that Snowmass participants will take time, together with our colleagues from around the globe, to reflect on the role of American particle physics in the world. How might our relationship with CERN evolve to the benefit of all? How can we improve our standing as a reliable partner in international collaborations based abroad as well as in the US? How can we most effectively encourage promising international students to come to our institutions and contribute to US-based research programs? Can our community once again muster the courage to offer the world a compelling new accelerator project attuned to scientific imperatives and commensurate with our nation's history of ground-breaking research? Make no little plans!

Chris Quigg: Fermi National Accelerator Laboratory, Batavia, IL, USA 


\section{REFERENCES}

1. F. Gianotti and G. F. Giudice, "A roadmap for the future," Nat. Phys. 16, 997 (2020).
2. C. Quigg, "Dream Machines," Rev. Accel. Sci. Tech. 10, 3 (2019). 3. C. Quigg, Perspectives and Questions: Meditations on the future of particle physics. 